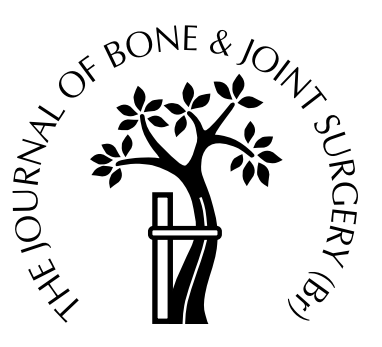

Ankle fractures in diabetics

\title{
COMPLICATIONS OF SURGICAL MANAGEMENT
}

\author{
R. G. McCormack, J. M. Leith \\ From the North West Orthopaedic and Sport Medicine Centre, \\ New Westminster, Canada
}

T o determine the relative risk of complications in treating ankle fractures in patients with diabetes, we compared the results of the management of displaced malleolar fractures in 26 patients with those of a matched group of non-diabetic patients, using a case-controlled study. The groups were matched for patient age, fracture type and treating surgeon.

The incidence of significant complications in diabetic patients was $42.3 \%$. By contrast, there were no complications in the matched group of non-diabetic patients. Of 19 diabetic patients treated surgically, six developed major complications including one case of malunion, one of necrosis of the wound edge requiring a flap, and two of deep sepsis. Two patients required amputation and both died. Diabetic patients with displaced ankle fractures treated non-operatively had a high incidence of loss of reduction and malunion but these caused few symptoms. In these patients, non-operative management may be preferable in view of the high risks of major complications after surgery and the acceptance of malunion by the older patient with lower demands.

J Bone Joint Surg [Br] 1998;80-B:689-92.

Received 16 December 1997 ; Accepted 3 March 1998

Diabetes mellitus affects approximately $6 \%$ of the world's population. Patients are at risk of a variety of systemic complications related to microangiopathy and peripheral neuropathy. ${ }^{1}$

Diabetics may sustain ankle fractures either as a result of a single traumatic event or, less commonly, because of repetitive forces on a neuropathic (Charcot) joint. The

R. G. McCormack, MD, FRCS C, Associate Professor

J. M. Leith, MD, Resident in Orthopaedic Surgery

Department of Orthopaedics, University of British Columbia, 10265 Richmond Street, New Westminster, British Columbia, Canada V5P $4 \mathrm{XI}$.

Correspondence should be sent to Dr R. G. McCormack.

(C)1998 British Editorial Society of Bone and Joint Surgery 0301-620X/98/48648\$2.00

VOL. 80-B, No. 4, JULY 1998 standard treatment of displaced intra-articular ankle fractures is open reduction and internal fixation. Given the associated microangiopathy, peripheral neuropathy and higher susceptibility to infection, it might be anticipated that the complication rates from surgery would be high. There is, however, little information on the outcome of surgical intervention in diabetics with displaced ankle fractures.

We performed a case-controlled study to compare the complication rates for ankle fractures in diabetics with a matched group of non-diabetic patients.

\section{Patients and Methods}

All patients with diabetes mellitus treated for malleolar fractures at our institution between April 1990 and January 1996 were identified from the database of the medical records. This refers to type-44 fractures in the comprehensive classification. ${ }^{2}$ Fractures of the distal tibia (plafond) and fractures of the talus were excluded. Tables I to III give details of the patients and the fractures with the method and outcome of treatment. Radiographs of the ankle before, during, and at completion of treatment were reviewed independently (Figs 1 to 4). Using the same hospital database, all non-diabetic patients treated for malleolar fractures over the same period of time were identified. A cohort was randomly selected which was matched

Table I. Details of the diabetic patients and the controls

\begin{tabular}{lll}
\hline & $\begin{array}{l}\text { Diabetic group } \\
(\mathbf{n = 2 6})\end{array}$ & $\begin{array}{l}\text { Non-diabetic control group } \\
(\mathbf{n = 2 6 )}\end{array}$ \\
\hline Mean age in years & 61 & 61 \\
Age range in years & 43 to 78 & 40 to 77 \\
Male & 8 & 5 \\
Female & 18 & 21 \\
\hline
\end{tabular}

Table II. Fracture patterns in the diabetic and control groups

\begin{tabular}{lcc}
\hline Fracture pattern & $\begin{array}{l}\text { Diabetic group } \\
(\mathbf{n = 2 6})\end{array}$ & $\begin{array}{l}\text { Non-diabetic } \\
\text { control group } \\
(\mathbf{n = 2 6})\end{array}$ \\
\hline Unimalleolar & 5 & 0 \\
Bimalleolar & 9 & 10 \\
Trimalleolar & 12 & 16 \\
\hline
\end{tabular}


R. G. MCCORMACK, J. M. LEITH

Table III. Incidence of complications in the diabetic and control groups

\begin{tabular}{llll}
\hline & $\begin{array}{l}\text { Diabetic group } \\
\begin{array}{l}\text { Operative treatment } \\
(\mathbf{n = 1 9 )}\end{array}\end{array}$ & $\begin{array}{l}\text { Non-operative treatment } \\
(\mathbf{n = 7 )}\end{array}$ & $\begin{array}{l}\text { Non-diabetic control group } \\
(\mathbf{n}=\mathbf{2 6})\end{array}$ \\
\hline Complication & & 0 & 0 \\
$\quad$ Wound edge necrosis & 1 & 0 & 0 \\
Deep wound sepsis & 2 & 5 & 0 \\
Malunion & 1 & 0 & 0 \\
Amputation followed by death & 2 & $5(71.4)$ & 0 \\
Total $(\%)$ & $6(31.6)$ & & \\
\hline
\end{tabular}

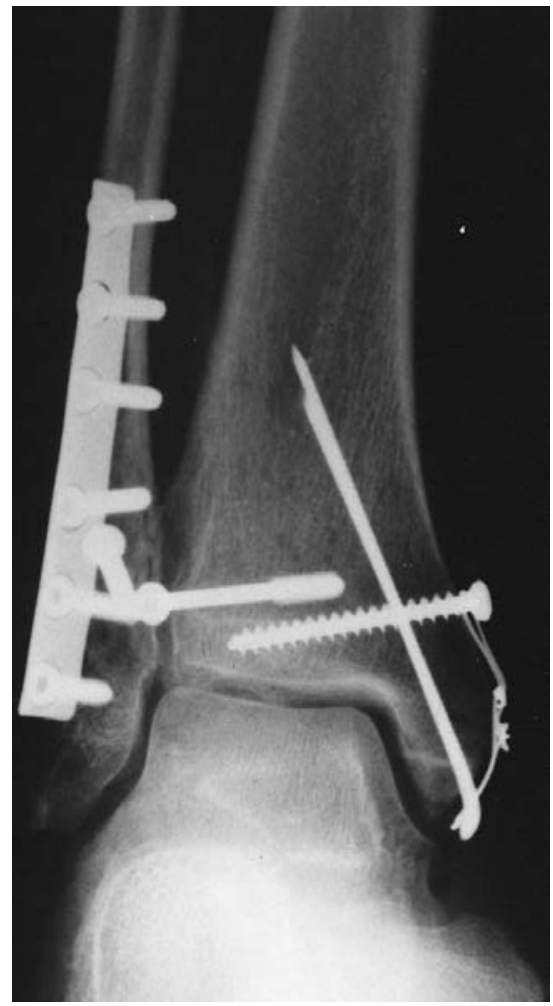

Fig. 1

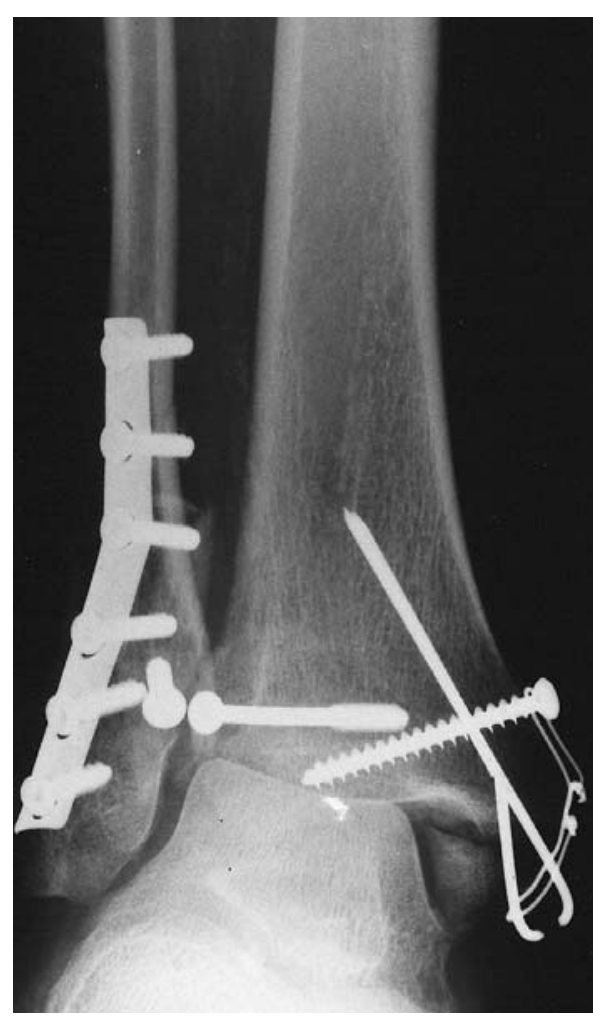

Fig. 2

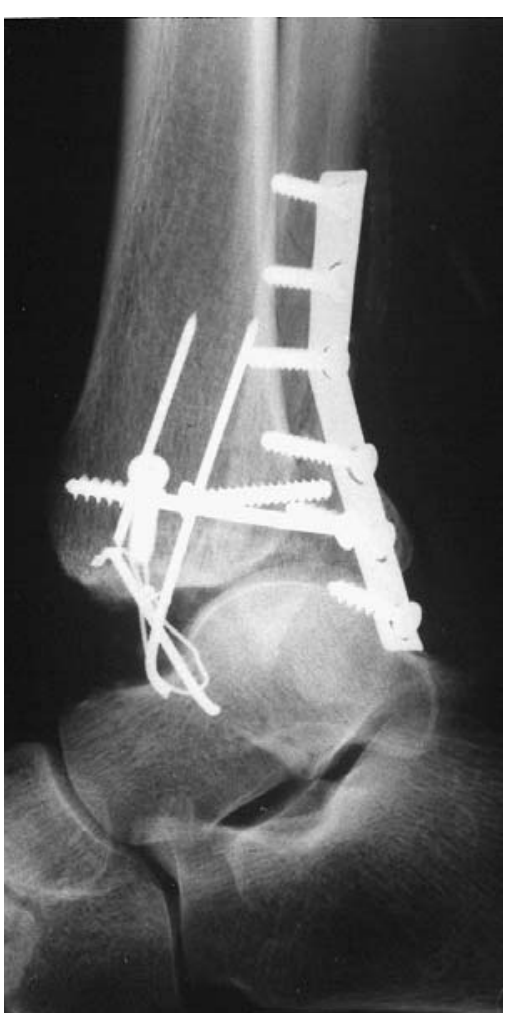

Fig. 3

Figure 1 - Anatomical reduction in a 57-year-old patient with diabetes. Figures 2 and 3 - Loss of reduction associated with wound breakdown and deep infection.

for patient age, fracture type and treating surgeon. The hospital records and radiographs for this control group were then analysed similarly to those of the diabetic group.

The decision to treat an ankle fracture in diabetic patients, by operation or not, was not randomised, but based on assessment by the surgeon. Generally, the nonoperative approach was selected in patients with poor health or low functional demands, in whom the surgical risk was not felt to be justified. Surgical treatment was carried out according to AO principles.

We used Fisher's exact test to assess the statistical significance of the differences in the incidence of major complications between the two groups, and the difference in outcome between the diabetics treated conservatively or by operation. The influence of insulin dependence and complications on clinical outcome was also assessed.

\section{Results}

We identified 26 patients with diabetes mellitus and malleolar fractures who were matched with 26 non-diabetic control subjects with similar injuries. Details of the two groups are given in Table I.

Of the diabetic group, 13 were insulin-dependent and 13 were controlled by diet and/or oral hypoglycaemic agents. The mean duration of their illness before presentation was 18.7 years ( 2 to 43 ).

There were five unimalleolar, 19 bimalleolar and 28 trimalleolar fractures. The distribution in both groups is outlined in Table II.

A minor complication of treatment was defined as superficial infection or necrosis of the edge of the wound and a major complication as deep infection, necrosis of the edge 


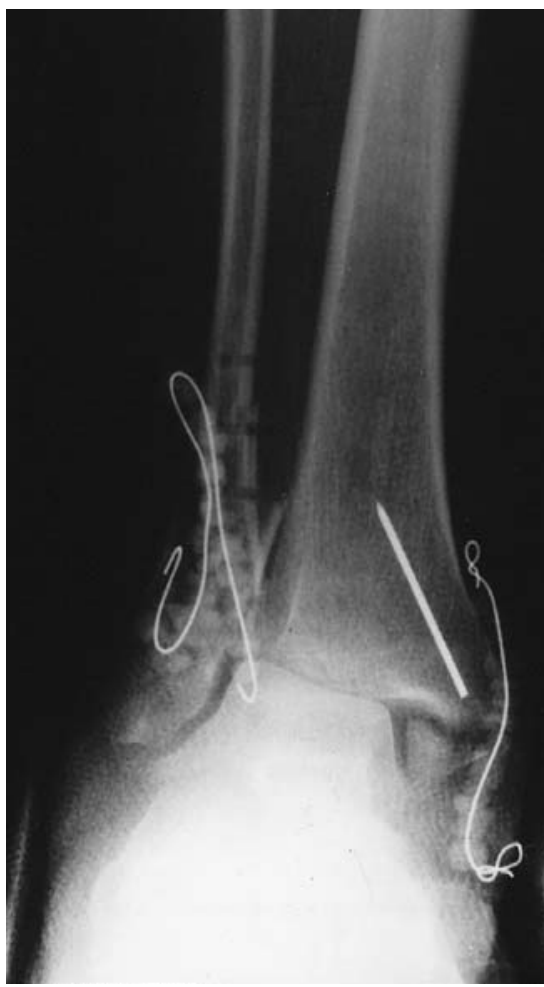

Fig. 4

Ankle of same diabetic patient after debridement and insertion of antibiotic beads.

of the wound or dehiscence requiring surgical intervention, amputation, malunion, or death. We excluded postoperative erythema not requiring treatment.

The total incidence of complications in the two groups is outlined in Table III. In the diabetic group, 11 of 26 patients developed a complication compared with none in the control group. This was statistically significant (Fisher's exact test, $\mathrm{p}=0.001)$.

Of the 19 operatively treated diabetics, five developed significant wound problems. These included necrosis of the edge of the wound requiring local flap closure in one patient, deep wound infection with septic arthritis in two and fulminant infection leading to amputation and death in two.

There was no statistically significant difference between the insulin-dependent and non-insulin-dependent diabetics with regard to the overall complication rate, but four of the five operatively-treated diabetic patients who developed wound problems, and both patients who died, were insulindependent. We could not detect any significant relationship between the presence of clinically recognised peripheral neuropathy or angiopathy and the likelihood of developing a complication.

The ankle fractures in diabetic patients treated without operation had no skin or soft-tissue problems. There was some loss of reduction and malunion in five of seven patients. These malunions caused few symptoms and all patients had a functional lower limb.

\section{Discussion}

Ankle fractures in diabetic patients treated by operation have a high incidence of serious wound complications, while those treated without operation are likely to develop a malunion. This is consistent with the results of other studies, suggesting that diabetes mellitus is a risk factor for complications after ankle fracture. ${ }^{3-5}$ The case-controlled design of our study defines this risk as a complication rate of $42 \%$ in diabetics against $0 \%$ in a matched group of non-diabetics. This includes a mortality of $8.5 \%$ and a rate of deep infection of $17 \%$. The design of the study makes the presence or absence of diabetes mellitus the variable, with controls for other major risk factors (patient age, fracture type and treating surgeon). The rate of surgical complication was unacceptably high despite the fact that the 'best risk' patients were selected for surgical intervention.

Diabetic patients who present with a traumatic ankle fracture are at a high risk of developing complications. The difference is all the more significant given that there was a trend to more complicated fractures in the control group with a greater proportion of both trimalleolar and bimalleolar fractures. The absence of complications in the group of non-diabetic patients matched for age, treating surgeon, and fracture type, suggests that the difference depends on patient factors rather than the institution or treating surgeon.

The aetiology of this high complication rate is multifactorial. Diabetic patients have an increased susceptibility to wound infections. They also have impaired wound healing from micro- and macroangiopathies. Impairment of nociception and proprioception can lead to delays in recognising pressure sores and ulceration from the plaster cast as well as inappropriate weight-bearing before union resulting in malunion.

In the fractures treated without operation in our series there were no problems related to the cast, but Zinar and Brown ${ }^{4}$ reported a $50 \%$ incidence of skin breakdown in diabetics with ankle fractures treated in a cast. He recommended open reduction and internal fixation if a peripheral neuropathy was present. His surgical complication rate was $40 \%$ which makes this conclusion difficult to justify. The risk of malunion and plaster sores should be minimised by regular radiological assessment and changes of the casts which should be suitably padded and provide widely distributed support.

Despite the fact that most diabetic patients treated without operation developed malunion, they had minor symptoms and all had a functional lower limb. It should be noted that our main focus in this report was on the rates of surgical complications and we did not perform formal outcome assessments of function.

There is a very high incidence of operative complications in diabetic patients with ankle fractures. In the older, diabetic patient with lower demands, especially if insulin- 
dependent, it may be preferable to accept a loss of reduction and malunion rather than risk the potentially devastating complications associated with operative intervention. If surgical intervention is necessary, our results from this case-controlled study will allow more accurate prognosis and discussion of the surgical risks with the patient.

No benefits in any form have been received or will be received from a commercial party related directly or indirectly to the subject of this article.
References

1. Leslie RDG, Robbins DC. Diabetes: clinical science in practice. Cambridge University Press, 1995.

2. Muller ME, Allgower M, Schneider R, Willenegger H. Manual of internal fixation techniques recommended by the AO Group. Third ed. Heidelberg, New York: Springer-Verlag, 1990:148-9.

3. Kristiansen B. Results of surgical treatment of malleolar fractures in patients with diabetes mellitus. Dan Med Bull 1983;30:4,272-3.

4. Zinar DM, Brown IC. Complications following treatment of acute ankle fractures in diabetic patients. Annual Meeting of the Orthopaedic Trauma Association Los Angeles, 1994.

5. Low CK, Tan SK. Infection in diabetic patients with ankle fractures. Ann Acad Med Singapore 1995;24:353-5. 